\title{
The structure of the initial cohort: Evidence from gating
}

\author{
LORRAINE K. TYLER \\ Max Planck Institut fur Psycholinguistik, Nijmegen, The Netherlands \\ and MRC Applied Psychology Unit, Cambridge, England
}

\begin{abstract}
Subjects heard successive fragments of words both in and out of context, and after each fragment they wrote down the word they thought they were hearing. Responses were analyzed for word frequency, length, and compatibility with both contextual constraints and the sensory input. These responses, pooled across subjects, enabled us to evaluate a number of claims concerning the use of top-down and bottom-up information in the process of word recognition. The analyses show that, in the process of recognizing a spoken word, subjects initially produce a large number of responses compatible with the sensory input. This set diminishes in size as more of the word is presented. The rate at which responses drop out of the initial set differs, depending on whether words are heard in isolation or in context. These properties of the elicited responses are compatible with claims made by the cohort model for the integration of top-down and bottomup information in the process of recognizing a spoken word.
\end{abstract}

It has recently been claimed that the recognition of a spoken word involves the activation of a large set of word candidates which gradually diminishes in size until only a single member remains (Marslen-Wilson \& Tyler, 1980; Marslen-Wilson \& Welsh, 1978). This 'word-initial cohort' is created from some minimal sensory inputperhaps equivalent to the first one or two phonemes of a word-and its initiation is not influenced by the context in which the word is heard. The number of word candidates in the cohort diminishes over time as more and more of them fail to match both contextual requirements and the accumulating sensory input. When there is only a single member of the cohort remaining, the listener recognizes the word (Marslen-Wilson \& Tyler, 1980; MarslenWilson \& Welsh, 1978; Tyler \& Wessels, 1983).

In this paper, I will assume that the cohort theory is correct in its claim that, in the process of recognizing a word, a set of word-candidates becomes activated. I will test certain predictions that this and, where possible, other theories make about these candidates-both about the characteristics of the initial set and the ways in which they change over time as a function of the contribution of sensory input and contextual constraints.

The cohort theory makes a number of specific predictions about factors influencing the initial construction of the cohort and its changing structure over time. Some of these predictions have been evaluated in previous research (Grosjean, 1980; Salasoo \& Pisoni, in press). One major theoretical issue concerns the composition of the initial

\footnotetext{
I wish to thank William Marslen-Wilson, Herb Clark, and two anonymous reviewers, for helpful discussions on the data and on the manuscript, and Jeanine Wessels, for carrying out the experiment and endlessly counting words. The author's mailing address is: MRC Applied Psychology Unit, 15 Chaucer Road, Cambridge, England.
}

cohort. Are all of the word candidates consistent with a given segment of speech activated, irrespective of their frequency, contextual appropriateness, and length? Grosjean (1980) has argued that they are not. He claims that the prior context also affects the composition of the cohort. Context reduces the size of the initial set and constrains the type of candidates activated. Grosjean also claims that word frequency plays an important role in the cohort, with members being organized or weighted according to frequency. Consequently, high-frequency words are more active members of the cohort and are recognized earlier than low-frequency words.

Salasoo and Pisoni (in press) take a position which is more consistent with the cohort theory. They propose that the cohort is initiated by a 'reliable' segment of speechabout the first $150 \mathrm{msec}$ of a word. When listeners hear much smaller segments than this, candidates can be proposed on the basis of top-down knowledge sources.

The other major issue concerns the process of narrowing down the cohort to a single member. Both Grosjean (1980) and Salasoo and Pisoni (in press) concur with the cohort model's claim that candidates drop out of the initial pool as they fail to meet the requirements imposed by the accumulating sensory input and contextual constraints. In contrast to this, Forster (1981), Seidenberg, Tanenhaus, Leiman, and Bienkowski (1982), and Swinney (1979) have claimed that the process of recognizing a word is totally independent of context. Context has an effect only after a word has been recognized. On a strong interpretation of this view, words should drop out of the cohort only as they become incompatible with the bottomup information-whether they are heard in isolation or in context.

What specific predictions does the cohort theory make about the factors influencing the construction of the ini- 
tial cohort and the way its size and composition change over time?

(1) According to the theory, all word candidates that match some initial segment of speech become active. This initial fragment has been estimated at about $150 \mathrm{msec}$ (Marslen-Wilson, $1980^{1}$; Salasoo \& Pisoni, in press). After the initial cohort has been defined, contextual influences come into force. Therefore, there should be a moment in time at which all members of the initial cohort are active-whether or not they are contextually appropriate. This point should be followed by the continuous assessment of all active word candidates to ensure that only those which match both the accumulating sensory input and the context remain in the cohort. This predicts that there will be a discontinuity between the point at which all word candidates match the sensory input, although some may be incompatible with the context, and a point at which all word candidates are compatible with both sources of processing information. If, on the other hand, context works in advance of the sensory input to restrict the initial set (e.g., Grosjean, 1980), then only word candidates that are contextually appropriate will ever be activated.

(2) Although word frequency plays an important role in many models of lexical access (e.g., Bradley, 1978; Forster, 1976; Glanzer \& Ehrenreich, 1980; Morton, 1969; Whaley, 1978), it has not yet been incorporated into the cohort model. Indeed, the finding that highfrequency words are recognized faster than low-frequency words (Foss, 1969; Gordon, 1983; McCusker, HolleyWilcox, \& Hillinger, 1979) or with less sensory input (Grosjean, 1980) potentially poses problems for the cohort theory. The amount of sensory input needed to recognize a word should be a function of the structure of the cohort of which it is a member, irrespective of its frequency or the frequency characteristics of the other members of the set. For frequency effects to be compatible with the cohort theory (as presently stated), they have to be located after a word has been recognized (defining "recognition" as that point at which a word is the only remaining member of its original cohort (cf. MarslenWilson \& Welsh, 1978). Once a word has been recognized, its frequency might affect, for example, the speed with which lexical decisions about the word can be made, or its integration with the context.

This predicts that a word's frequency should not affect its recognition point. Unfortunately, stadies showing either that high-frequency words are responded to faster than low-frequency words (McCusker et al., 1979) or that recognition occurs earlier to high-than to low-frequency words (Grosjean, 1980) do not test this prediction. They do not look at the relationship between the point at which the word is recognized and the structure of the cohort at that point. It might just be the case that, in general, lowfrequency words require more acoustic-phonetic input before their recognition point is reached than do highfrequency words. This would be an explanation in terms of the cohort structure of the language rather than of word frequency.

Only one experiment provides appropriate data with which to evaluate this prediction, although it was not set up with this issue in mind. Tyler and Wessels (1983) found that both high- and low-frequency words were recognized at the point at which the word was the only surviving member of its original cohort. ${ }^{2}$ This suggests that even if a word is highly frequent, it will not be recognized before it becomes the only remaining candidate.

The cohort theory also predicts that frequency per se should not determine membership of the initial set of word candidates. Words that match the initial segment should be activated, irrespective of frequency. This prediction will be tested here.

(3) If, as the cohort theory predicts, the initial set is activated purely on the basis of the sensory input, then, given a particular input, its size should be constantirrespective of the context in which the word is heard. If, however, there is contextual preselection (Grosjean, 1980; Morton, 1969) in which only contextually appropriate word candidates ever become active, the size of the initial cohort will vary according to the strength of contextual constraints.

(4) Context should have a major effect on the rate at which the pool of word candidates is reduced over time. When words are heard in isolation, word candidates can drop out of the initial pool only as their internal specifications diverge from the accumulating sensory input. But when they are heard in utterances, word candidates also drop out of the set if they are contextually inappropriate. However, if context has an effect only after a word has been identified on the basis of the sensory input (Forster, 1981; Seidenberg et al., 1982; Swinney, 1982), then it should not affect the drop-off rate.

In order to study the influence of these factors on the structure of the cohort, I used the gating task that Grosjean (1980) developed to investigate spoken word recognition processes. In this task, listeners hear successively larger fragments of target word. After hearing each fragment, the subjects indicate which word they think is being presented, either by writing down the word (e.g., Cotton \& Grosjean, 1984; Grosjean, 1980; MarslenWilson, 1984; Salasoo \& Pisoni, in press; Tyler \& Wessels, 1983) or by naming it out aloud (e.g., Nooteboom \& Truin, 1980; Tyler \& Wessels, 1984).

The gating task is one of the few experimental tasks that provides information about the characteristics of the cohort structure for a word at any particular point in its processing history. To use the task in this way, we assume, in common with other researchers (e.g., Grosjean, 1980; Salasoo \& Pisoni, in press), that the set of word candidates produced at each segment, pooled across subjects, enables us to estimate the general properties of the set of words active at each segment for an individual listener. Given this assumption, we can determine the properties of the initial set and chart its progress over time. 
We can thus see how subjects' responses change as they become incompatible with the accumulating sensory input and/or contextual constraints.

The validity of this assumption can be determined only by comparing data from the gating task with those from other paradigms. We know that the gating task provides the same estimates of word recognition as a number of on-line tasks (cf. Grosjean, 1980; Marslen-Wilson, 1984), but no other task currently in use also provides an estimate of the structure of a cohort.

The data I will be analyzing here were obtained in an experiment investigating the extent to which syntactic and semantic constraints reduce the sensory input needed for spoken word recognition. Using a gating task, listeners heard successive increments of target words that appeared either in isolation or in sentential contexts in which the strength of syntactic and semantic constraints was covaried. The amount of sensory input required for recognition of targets appearing in the various conditions has been reported elsewhere (Tyler \& Wessels, 1983). In this paper, I will analyze the words subjects produce before they correctly recognize the targets. I will assume that these sets of words reflect the members of activated cohorts, and will analyze their structure, to determine what this reveals about the properties of the cohort when it is initially activated, and the factors that affect its change over time.

\section{METHOD}

\section{Subjects}

Sixty native speakers of Dutch participated in the study.

\section{Materials}

Target words consisted of 25 infinitive verbs, each with an initial cohort (based on the first two phonemes) of over 60 members, at least 20 of which were infinitive verbs. These counts are a conservative estimate of the size of each cohort since they did not include inflected or derived forms. ${ }^{3}$ For each target verb, I constructed four sentence pairs with the target in the second sentence of each pair. In two pairs, the material was semantically anomalous and could not semantically facilitate identification of the target. In the other two pairs, the material preceding the target provided a minimal semantic context for the target. A pretest, using an auditory cloze procedure, established that the semantic contexts in these conditions were indeed only minimally constraining. In this pretest, listeners heard the first $100 \mathrm{msec}$ of each target in the four context conditions. This restricted the set of words likely to be produced and provided a more conservative test of a target's predictability than if none of the target had accompanied the context sentences.

For the pretest, four versions of the materials were constructed, with conditions pseudorandomly distributed within a version. Eight subjects were tested on a version. Listeners responded with an appropriate continuation for each sentence pair. The following scoring procedure was used: $1=$ identity with the target, $2=$ synonym of target, $3=$ related to target, $4=$ contextually appropriate but unrelated to target, and 5 $=$ contextually inappropriate and unrelated to target.

Two independent judges scored responses according to the above criteria. The mean rating for the two semantically normal conditions were 3.95 and 3.96 , indicating that although responses were contextually appropriate, subjects rarely produced the target word itself. For the two semantically anomalous conditions, the ratings were 4.88 and 4.97 , showing that responses were unrelated to the intended target.

The syntactic constraints were manipulated by varying local restrictions on form class. The weak-syntactic-constraint condition placed minimal restrictions on the form class of the target-only certain in- flected forms of verbs were prohibited. In contrast, in the strongsyntactic-constraint condition (which was the most syntactically constraining that Dutch allowed), the target was preceded by the word $t e$, which can be followed only by an infinitive or by one of a small number of adjectives.

So there were four experimental conditions:

(1) Minimal semantic context/strong syntactic constraints

(2) Minimal semantic context/weak syntactic constraints

(3) Anomalous context/strong syntactic constraints

(4) Anomalous context/weak syntactic constraints

In a fifth condition, the target word appeared without any supporting context. An example of the materials (in Dutch) ${ }^{4}$ :

Target word: Profiteren

(a) Minimal sem/strong syn: De afspraak met de tandarts gaat niet door. Jan probeert te ....

(b) Minimal sem/weak syn: De afspraak met de tandarts gaat niet door. Jan kan....

(c) Anomalous/strong syn: De adem met de leugen schuift pas door. Het terras tracht te....

(d) Anomalous/weak syn: De adem met de leugen schuift pas door. Het terras wil ....

Target words spoken in a neutral carrier phrase ("The following word is . . .'") were recorded by a female native speaker of Dutch. Each target was excised from its carrier phrase, digitized at a sampling rate of $20 \mathrm{kHz}$, and segmented from the onset of the word into fragments, each of which increased in duration by $50 \mathrm{msec}$. So the first segment consisted of the first $50 \mathrm{msec}$ of the word, the second consisted of the first $100 \mathrm{msec}$, and so on until the whole word had been output. The number of segments for each word depended upon the total duration of the word.

These fragments were inserted into recordings of each of the four context conditions, and they were also used as stimuli in the no-context condition in which the segments were presented in isolation. So the same acoustic tokens appeared in all conditions.

Since each target word occurred in all five experimental conditions and each subject was intended to hear a word in a single condition, five versions of the materials were constructed. Each version contained five instances of each of the five conditions, with the five no-context items blocked at the beginning of a version, and the items in the other four conditions pseudorandomly distributed across each version. There were 25 filler items and 10 practice items. Twelve subjects were tested on each of the five versions. The successive presentation method was used with each subject's hearing increasingly larger fragments of each target. (Additional experimental details can be found in Tyler \& Wessels, 1983.)

\section{Procedure}

The 60 subjects were tested in groups of 4 . They were told to write down after every fragment the word they thought they were hearing, and then to decide how confident they were on a scale of 1-10, with 10 being absolutely confident and 1 being completely unsure.

\section{RESULTS AND DISCUSSION}

I tabulated subjects' word choices at each segment in each of the five conditions. This set of experimentally induced word choices will be called the elicited cohort. The word frequency, syllable count, syntactic and semantic appropriateness, and form class of each word choice were recorded. It was impossible to make any meaningful analysis of form class because a high proportion of word 
choices were syntactically ambiguous. Therefore, I will not discuss that aspect of the data. ${ }^{5}$

\section{Factors Influencing the Initiation of a Cohort}

(1) Amount of sensory input needed for correct identification of the first phoneme of a word. The question here is whether context influences the initiation of a cohort by facilitating identification of the initial phoneme of a word. Grosjean's (1980) data suggest that it does. $\mathrm{He}$ found that when words were heard in isolation, only $23 \%$ of responses began with the correct consonant after $30 \mathrm{msec}$ of the signal, whereas $78 \%$ correct identification was achieved when the same fragment was heard in a meaningful context. In the present study, with a 50-msec first gate, there was no difference in the accuracy with which the first phoneme was identified in the five experimental conditions. It was correctly identified $62 \%$ of the time in the isolation condition, $68 \%$ in both minimal semantic conditions, and $63 \%$ in each of the anomalous semantic conditions $(F<1)$. The incorrect phonemes that were produced shared certain acoustic-phonetic features with the target phoneme. For example, the phoneme $/ \mathrm{v} /$ was frequently misperceived as /f/. These results are similar to those reported by Salasoo and Pisoni (in press). They also found that the proportion of incorrect responses that were compatible with the acoustic-phonetic input did not differ as a function of context.

These data are compatible with the claim that the initiation of a cohort is based only upon the sensory input and is unaffected by prior context. This is why the majority of initial phonemes are compatible with the sensory input-even when they are not the target phoneme, and why the presence of a meaningful context did not facilitate identification of the first phoneme in the first $50 \mathrm{msec}$. If context had functioned predictively to narrow down the potential cohort in advance of any sensory input (e.g., Grosjean, 1980; Morton, 1969), we would have expected to find more correct identifications in the context conditions than in isolation.

The present data presumably differ from Grosjean's because the strength of contextual constraints on the target varied considerably in the two studies. ${ }^{6}$ Acknowledging the possibility of contextual effects here does not imply that context necessarily works in advance of any sensory input, but merely that when contextual constraints are strong, word candidates (based on the bottom-up input) can be evaluated more effectively for their contextual suitability and those which are inappropriate will drop out of the pool.

Grosjean's data are also unusual in the low identification rate for 30 -msec segments in isolation (23\%). This differs from my results and from the results reported by Amerman and Parnell (1981), who found 95\% accuracy with a 20 -msec window. The discrepancy cannot simply be due to the different initial phonemes used in the three studies. Although Grosjean used a variety of initial consonants (some of which were common to all three experiments), accurate identification was never higher than $50 \%$.

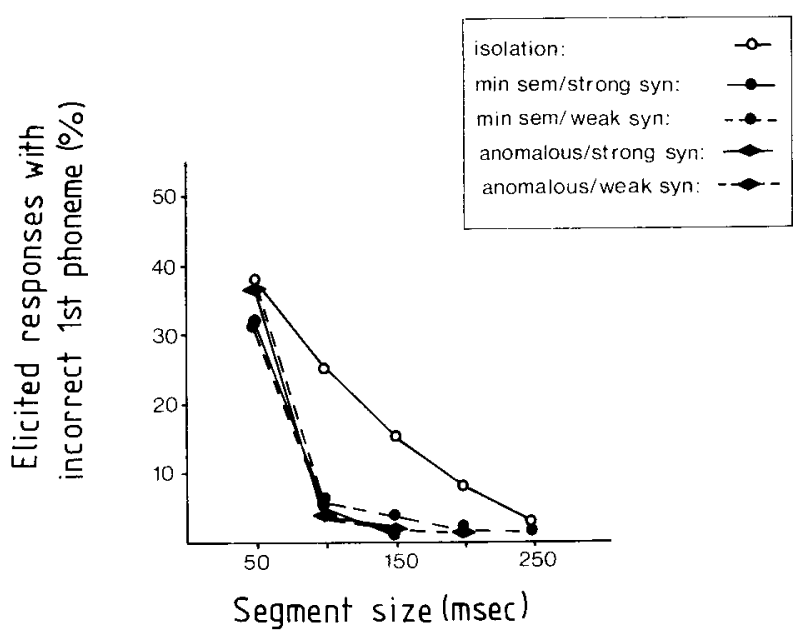

Figure 1. The percentage of elicited responses with incorrect first phonemes at segments 1-5 in each experimental condition.

In my data, differences between the isolation condition and the context conditions emerge by the second gate $(100 \mathrm{msec})$. For all four contexts (see Figure 1), there are few responses containing the wrong first phoneme by the second gate, and this remains unchanged across gates $(\mathrm{F}<1)$. In contrast, in the isolation condition there are still a large number at the second gate, and this gradually decreases across gates [Gates 2-5 $\times$ items: $F(3,48)=$ $9.926, \mathrm{p}<.001]$. One explanation of this result is that the context preceding the target segment helps the listener adjust to the speaker's voice and therefore increases the efficiency with which the speech input can be interpreted. But since, in all conditions, the target was excised from the context in which it was originally spoken (i.e., the neutral carrier phrase), it must be the general properties of the speaker's voice which have this effect.

Fifty milliseconds of a target in isolation was less likely to evoke a response (whether correct or incorrect) than when the same fragment occurred in a context. There were $32 \%$ nonresponses in the isolation condition. This compares with $15 \%$ in both the minimal semantic/strong syn$\operatorname{tax}[t(16)=3.274, p<.01$, two-tailed] and weak syntax $[\mathrm{t}(16)=3.139, \mathrm{p}<.01$, two-tailed $]$ conditions, and with $22 \%$ in the anomalous/strong syntax condition [ $\mathrm{t}(16)=$ $1.474, \mathrm{p}<.05]$ and $19 \%$ in the anomalous/weak syntax condition $[\mathrm{t}(16)=2.746, \mathrm{p}<.05$, two-tailed]. Thus, contextual constraints facilitate the initiation of a lexical hypothesis after only $50 \mathrm{msec}$ of the speech input, even though they may not help in the correct identification of the initial phoneme. This suggests that an acousticphonetic fragment heard in context is a more appropriate stimulus with which to gain access to the mental lexicon.

(2) The size of the initial cohort. Grosjean (1980) has reported that the size of the initial cohort is larger for acoustic fragments heard in isolation ( 7.5 candidates) than in context, and that a weak context elicits a larger set (6.08) than a strong context (4.73). He argues from this that context serves to narrow down the set of word candidates to those that are contextually appropriate before 
the subject has heard any of the sensory input. In the present study, however, the mean number of word candidates produced at the first gate (after $50 \mathrm{msec}$ ) did not vary across conditions. Isolated fragments elicited a mean of 7.1 responses, meaningful contexts elicited 7.9 , and anomalous sentences $8.5(\mathrm{~F}<1)$. These data suggest that context does not constrain the size of the initial set in advance of the sensory input.

In fact, these two sets of data are not inconsistent. The pool of elicited responses is substantially reduced only when there are very strong semantic constraints (in Grosjean's study). When semantic context is relatively weak, then in both studies there is only a small reduction in the size of the initial set compared with that of the isolation condition. Therefore, it appears that only strongly constraining semantic contexts significantly reduce the size of the initial cohort (as reflected in the gating task). Once again, this does not necessarily mean that context works predictively to constrain the set in advance of any sensory input. Strong contextual constraints might just be more effective in reducing the size of a cohort that is activated by the bottom-up input.

(3) Syllable count of elicited word candidates. When subjects heard fragments of multisyllabic words in isolation, $66 \%$ of their responses were monosyllabic words. Irrespective of the number of syllables in the target word, twice as many monosyllabic word candidates were produced as bisyllabic words, and almost no three- or four-syllable words (see Table 1). This is reflected in the results of ANOVAs performed on the percentages of elicited word candidates of $1,2,3$, and 4 syllables as a function of the number of syllables in the target word. In all cases, there were more monosyllabic than multisyllabic words [two-syllable targets, $\mathrm{F}(3,15)=37.18$, $\mathrm{p}<$ .001 ; three-syllable targets, $\mathrm{F}(3,12)=9.14, \mathrm{p}<.001$; four-syllable targets, $F(3,15)=6.06, p<.01]$. This preference for monosyllabic word candidates in the isolation condition diminished at different rates, depending on the number of syllables in the target word (see Appendix).

Table 1

Percentage of Elicited Responses of 1, 2, 3, or 4 Syllables in Length at the First Gate as a Function of Number of Syllables in Target and as a Function of Context

\begin{tabular}{lrrrrr}
\hline & \multicolumn{6}{c}{ Number of Syllables in Elicited Responses } \\
\cline { 2 - 6 } \multicolumn{1}{c}{ Context } & 1 & 2 & 3 & 4 & Mean \\
\hline \multirow{5}{*}{ Two-Syllable } & Targets \\
Isolation & 63 & 37 & 3 & 0 & 1.3 \\
Anomalous & 46 & 42 & 12 & 0 & 1.9 \\
Minimal Semantic & 22 & 60 & 17 & 1 & 1.95 \\
& Three-Syllable & Targets & & \\
Isolation & 69 & 20 & 11 & 0 & 1.2 \\
Anomalous & 27 & 36 & 31 & 6 & 2.3 \\
Minimal Semantic & 19 & 24 & 46 & 11 & 2.8 \\
& Four-Syllable Targets & & \\
Isolation & 66 & 16 & 16 & 2 & 1.2 \\
Anomalous & 31 & 31 & 21 & 9 & 2.3 \\
Minimal Semantic & 13 & 31 & 35 & 21 & 2.8 \\
\hline
\end{tabular}

Table 2

Percentage of Syntactically Inappropriate Responses Produced in the Four Context Conditions

\begin{tabular}{lcc}
\hline & \multicolumn{2}{c}{ Context } \\
\cline { 2 - 3 } Syntax & Anomalous & Minimal Semantic \\
\hline Strong & 15.4 & 4.4 \\
Weak & 4.8 & 1.2 \\
\hline
\end{tabular}

There was no such preference for monosyllabic words when fragments were heard in context. As Table 1 shows, in the minimal semantic conditions, only a small percentage of monosyllabic words were produced for all target words. ${ }^{7}$ There was also an increased probability of producing word candidates in the first gate, which contained the same number of syllables as the target. Sixty percent of word candidates at the first gate were bisyllabic when the target was bisyllabic, $46 \%$ were trisyllabic when the target was trisyllabic, and $21 \%$ consisted of four syllable words when the target was also four syllables long. This contrasts with the isolation condition, in which the proportion of word candidates at the first gate sharing the same number of syllables as the target was $37 \%$ for bisyllabic targets, $11 \%$ for trisyllabic targets, and $2 \%$ for four-syllable targets (see Table 2).

The data for the anomalous context conditions ${ }^{8}$ lies in between the isolation condition and the minimal semantic conditions. Fewer monosyllabic words were produced than in the isolation condition, but more than in the meaningful context conditions.

This means that a sentential context increases the probability that an elicited word will be the same length as the target, even after only $50 \mathrm{msec}$ of speech, and with each successive gate the probability grows even larger. In the minimal semantic conditions, most word candidates were the same length as the target by the second or third gate. This contrasts with the isolation condition, in which large numbers of elicited words matched the length of the target only by the fifth gate (see Appendix).

I conclude from this that the utterance preceding the speech fragment must provide the listener, directly or indirectly, with cues about the length of the target word. The prosodic contour is one plausible source of direct cues since it allows listeners to anticipate where stressed syllables will occur (e.g., Shields, McHugh, \& Martin, 1974). Indirect cues may, for example, be syntactic in nature. If the target is constrained to be a verb in the infinitive form, then it must have at least two syllables (in Dutch). If, in addition, the first $50-100 \mathrm{msec}$ indicates that the word is prefixed, then the target must have at least three syllables.

The important issue is where, in the recognition process, prosodic or syntactic cues have an effect. There are two possibilities. Either the cues function predictively to reduce the set of potential word candidates or they operate upon an initial set activated on the basis of the sensory input. Although these data are not decisive, the results are more in favor of the latter possibility than of the former. If prosodic or syntactic constraints worked predictively, then there should have been no monosyllabic responses 
in the minimal-semantic/strong-syntax condition at the first gate, since the syntactic constraints require the target to be an infinitive verb and therefore to have at least two syllables (in Dutch). If, on the other hand, these constraints have an effect only after the cohort begins to be initiated by the sensory input, then we would expect to find a gradual concordance between the length of the targets and subjects' responses. Since this is what I did find, it suggests that there is an early integration of prosodic and syntactic information with the active members of the cohort.

(4) Frequency characteristics of elicited word candidates. I investigated two questions. First, does word frequency affect the composition of the initial cohort, or its changing structure over time? Second, does the context in which a fragment is heard affect the frequency characteristics of the word candidates produced?

Each elicited word candidate was categorized, where possible, as either low (1-10 per 720,000), medium (1130 per 720,000 ), or high ( 31 and above) frequency in the Dutch language (Uit den Boogaart, 1975). The proportion of word candidates in each category was calculated for each gate, in each of the five experimental conditions, and then entered into an ANOVA. The data for the sixth gate were omitted because there were too few word candidates.

In all experimental conditions, there were almost twice as many high-frequency words (63\%) as medium- $(19 \%)$ or low-frequency $(18 \%)$ words at the first two gates [gate $\times$ frequency, $F(8,640)=19.09, p<.001]$. This replicates Grosjean's (1980) finding that subjects produced more high- than low-frequency words, even when the target was of low frequency.

The preference for high-frequency words started to disappear by the third gate, and by the fifth gate $(250 \mathrm{msec}$ into the word) there were more low-frequency $(41 \%)$ than either medium $(31 \%)$ or high-frequency $(28 \%)$ words. ${ }^{9}$ These results are presented in Figure 2. The data have been collapsed across conditions since there was no difference between them $(F<1)$.

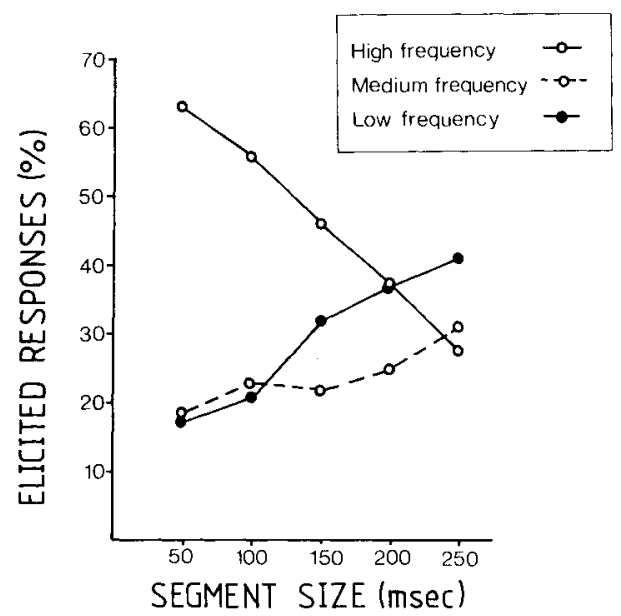

Figure 2. The percentage of high-, medium-, and low-frequency responses at segments $1-5$, collapsed across conditions.
There are a number of possible interpretations of this pattern. First, it could reflect production constraints. Since subjects can produce only one word at each gate, they have to select one word candidate from among all the active members of the cohort. One of the selection criteria might be that the more frequent a word, the more likely it will be chosen. This would not explain, however, why more low-frequency words are produced at later gates.

Second, the pattern of frequency effects could be due to high-frequency word candidates' having a lower initial activation "threshold." Assuming that all word candidates compatible with the sensory input are activated, those of higher frequency might become available for further analysis faster than words of low frequency. Words that are available soonest might be produced first in this task. ${ }^{10}$ If this is the correct explanation of the present frequency effects, then the cohort theory will need to be modified to include the concept of activation threshold (see Marslen-Wilson, 1984).

Third, the initial cohorts might have contained a large number of high-frequency words because more high- than low-frequency candidates were consistent with a given segment of speech. This is an explanation in terms of the structure of the cohort rather than word frequency per se. There is some evidence to support this in English, in which high-frequency words tend to be more similar to other high-frequency words ${ }^{11}$ than to low-frequency words (Landauer \& Streeter, 1973). Unfortunately, there is no equivalent data for Dutch.

Whatever the source of these frequency effects, the preference for high-frequency words lasts for only the first $150 \mathrm{msec}$. This implies that frequency effects are strongest under conditions of stimulus uncertainty. When listeners can clearly identify the beginning of the word, the frequency of word candidates plays a less important role. I will discuss the implications of these results in the conclusions section.

(5) Contextual appropriateness of word-candidates. The question here is whether context works predictively to partition the set of potential word candidates (Grosjean, 1980). If it does, then subjects should not produce any contextually inappropriate responses. If, however, context operates on a set initiated from the bottom up, we should find some contextually inappropriate responses in the early gates.

All word candidates produced at each gate in the four context conditions were evaluated to determine whether they satisfied the syntactic and semantic demands of the prior context. This was done by three native speakers working independently. Disagreements were discussed and resolved. First, the analysis of syntactic appropriateness (Table 2):

The number of syntactically inappropriate word candidates differs in the four context conditions $[F(3,48)=$ $5.833, \mathrm{p}<.001]$, with the largest number occurring in the anomalous/strong syntax condition. ${ }^{12}$ The difference between this and each of the other three conditions was significant $(\mathrm{p}<.01$ for all comparisons on NewmanKeuls). No other comparisons were significant. 
The larger number of syntactically inappropriate responses in the anomalous/strong-syntax condition was especially noticeable in the first gate. The percentage of syntactically inappropriate responses in the first gate in the anomalous/strong-syntax condition was $27 \%$ as opposed to $5 \%$ and $1 \%$ in the minimal-semantic/strong- and weak-syntax conditions, respectively, and $7 \%$ in the anomalous/weak-syntax condition.

Very few inappropriate words could have been produced in the weak-syntax conditions simply because almost all syntactic form classes were, by definition, permissible. What is of interest is the large difference between the two strong-syntax conditions. Many fewer syntactically inappropriate word candidates were elicited in a meaningful, as opposed to meaningless, context.

Secondly, the analysis of semantic appropriateness: Very few semantically inappropriate candidates were produced in the two minimal semantic conditions $-5.4 \%$ in the strong-syntax condition and $2.6 \%$ in the weaksyntax condition (this difference was not significant on the Newman-Keuls). Most semantically inappropriate responses occurred in the first few gates. Eight to nine percent of word candidates produced in the first three gates of the strong-syntax condition were semantically inappropriate, and $2 \%-4 \%$ were so in the weak-syntax condition. ${ }^{13}$ By the time the subjects had heard 250 msec of the word, they produced almost no semantically inappropriate responses.

These results are incompatible with any theory that claims that context acts predictively to constrain the initial set of word candidates. To be consistent with this claim, subjects should not have produced any contextually inappropriate responses. These data are, however, consistent with the cohort theory's claim that, when the cohort is in the process of being established, some word candidates will be contextually inappropriate.

\section{Narrowing Down the Initial Cohort}

According to the cohort theory, there should be fewer and fewer word candidates as segment size increases. For segments heard with no context, a mismatch with the accumulating sensory input is the sole criterion by which word candidates drop out of the pool. However, with a sentential context, contextual criteria can also be applied, and the initial cohort should be narrowed down more rapidly. Grosjean (1980) cites evidence in support of this general picture. He reports that top-down and bottom-up information interact in the process of reducing the size of the cohort.

To undertake a quantitative test of this claim, I calculated the reduction in the number of different word candidates produced at each gate in each of the five conditions. The reduction in the size of the set of elicited word candidates at each successive segment shows the differential rate at which the size of the cohort diminishes over time in the various conditions. These data are presented in Figure 3.

The isolation condition. The drop-off rate for the isolation condition is very different from those for all other conditions. For segments heard in isolation, there is only a very small reduction in the number of elicited word candidates for the first three gates. This means that few word candidates can be eliminated on the basis of the first $150 \mathrm{msec}$ of the sensory input. Perhaps this is because the first $150 \mathrm{msec}$ is the time period needed for setting up an initial cohort, as Marslen-Wilson (1980) and Salasoo and Pisoni (in press) have suggested. If so, we would not expect to find much of a reduction in the size of the cohort during this period.

After the 3rd gate, there is a slight increase in the number of word candidates which drop out of the cohort with each successive gate. This drop-off rate remains stable at a rate of about 0.8 word candidates per gate, until the 10 th gate, when it slows down again to 0.4 . Presumably, this steady decrease reflects the rate at which members of the cohort fail to meet the specifications of the accumulation sensory input.

Context conditions. The two minimal semantic context conditions differ from the isolation condition in both the earliness with which word candidates start to drop out of the pool and the rate at which they do so. Here there is a reduction of 2.5 word candidates by the fourth gate, but a reduction of only 0.3 word candidates at this point in the isolation condition. After a sharp drop-off in the number of word candidates (up to the fifth gate in the strong-syntax condition and one gate later in the weaksyntax condition), the size of the decrease diminished rapidly.

The data from the anomalous/strong-syntax condition are similar to those from the minimal-semantic conditions in showing a rapid acceleration in the drop-off rate of word candidates around the beginning of the word. Presumably, this is because word candidates are starting to drop out of the pool as they fail to satisfy the requirements imposed by strong syntactic constraints. In contrast, in the weak-syntax condition, there is hardly any reduction in the size of the cohort until $200 \mathrm{msec}$ of the signal has been heard.

What these results show is that when active members of the cohort can be evaluated only against the accumulating sensory input (the isolation condition), there is a steady decrease in the reduction in the size of the cohort throughout most of the word. However, when word candidates are also evaluated against contextual specifications (minimal semantic context conditions), there is a rapid reduction in the size of the cohort, reflecting the fact that many members of the original set are contextually inappropriate. The rate at which the size of the cohort is reduced slows down after approximately $250 \mathrm{msec}$ of the word has been heard. This is because an increasing number of subjects are beginning to identify the word around this point.

Finally, the cohort theory predicts that this drop-off in the number of word candidates continues until only a single word candidate remains, and it is at this point that the listener recognizes a word. The recognition point for a word is indeed closely related to the point at which only a single member remains of the original cohort (Tyler \& 

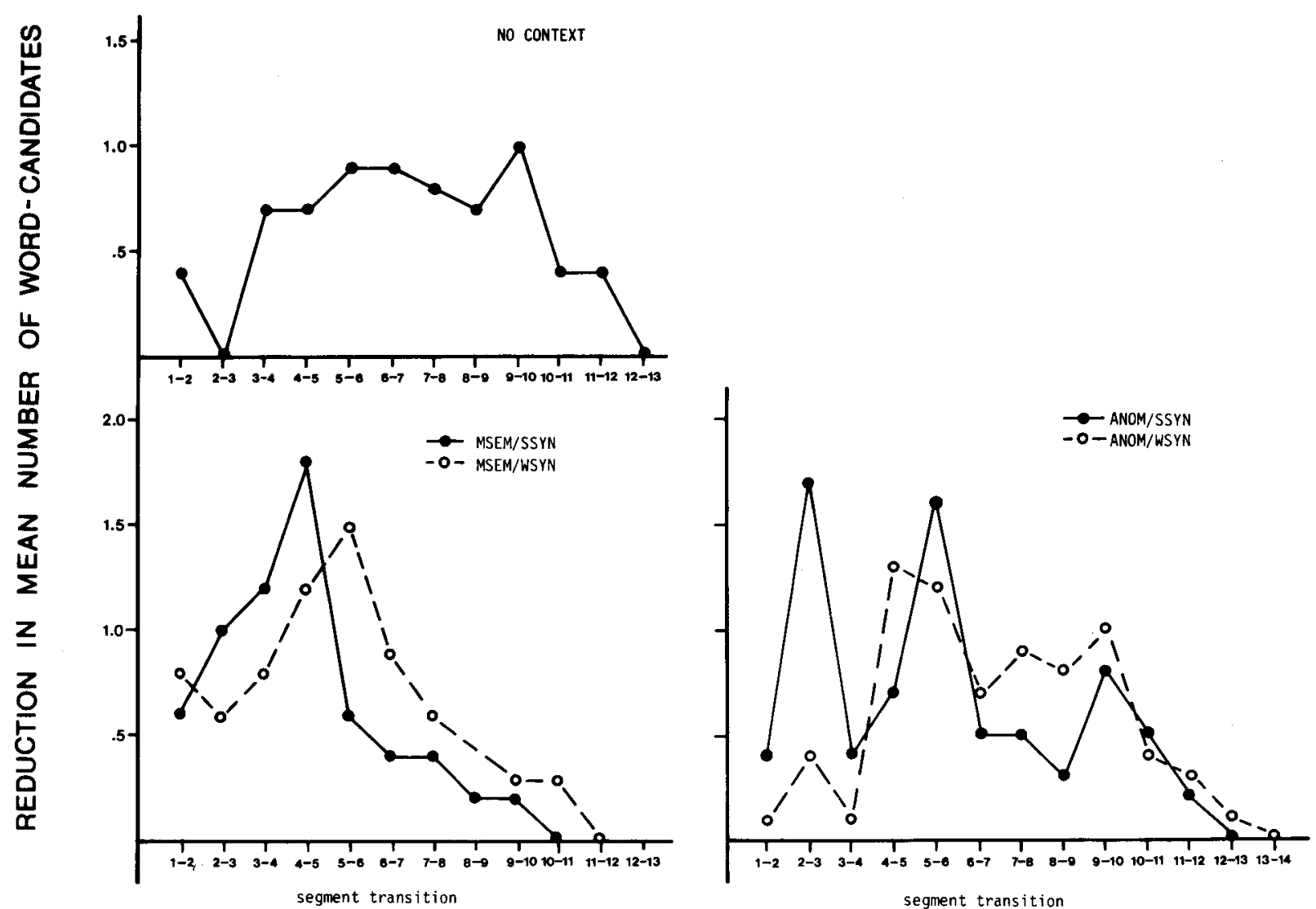

ANOM/SSYN $=$ Anomalous/strong syntactic constraints
ANOM/WSYN $=$ Anomalous/weak syntactic constraints
MSEM/SSYN $=$ Minimal semantic/strong syntactic constraints
MSEM/WSYN = Minimal semantic/weak syntactic constraints

Figure 3. Reduction in the mean number of responses elicited with increasing segment size for each condition.

Wessels, 1983). This process can also be seen in Figure 3. The mean recognition point (as calculated in the Tyler \& Wessels, 1983, study) for targets heard in isolation $(469 \mathrm{msec})$, for example, approximately corresponds to the 10 th gate. It is after this gate that we see a rapid decrease in the drop-off rate, because by that time almost all subjects have recognized the word. Similarly, in the two minimal semantic conditions, the drop-off rate peaks just before the mean recognition points ( 381 and $401 \mathrm{msec}$ for the strong- and weak-syntax conditions, respectively).

\section{CONCLUSIONS}

What conclusions can we draw about the initial cohort and the way its membership changes over time? How far do the results fit the predictions of the cohort model as originally stated, and how far should they cause the original claims to be modified?

\section{Bottom-Up Priority}

How do the data bear on the issue of the priority of bottom-up information? There is some evidence in its favor. Most responses were consistent with the sensory input, even when they were contextually inappropriate. In the anomalous/strong-syntax condition, for example, a large proportion of responses produced with a $50-\mathrm{msec}$ gate had the correct first phoneme, even though $27 \%$ of them were syntactically inappropriate.

However, not all responses with a 50-msec segment had the correct first phoneme ( $35 \%$ on average). Is this evidence against the claim for bottom-up priority? Only if we make two assumptions: first, that $50 \mathrm{msec}$ is always sufficient sensory input to unambiguously identify a phoneme, and second, that the cohort is established in the first $50 \mathrm{msec}$. If, however, we assume, along with Marslen-Wilson (1980) and Salasoo and Pisoni (in press), that approximately $150 \mathrm{msec}$ is needed to set up a cohort, this being the average amount of sensory input needed to reliably identify an initial segment (perhaps a diphone), then we would expect some proportion of responses in the first 150 msec to have the wrong initial segment.

The strongest evidence against the claim for bottom-up priority would be to find that a significant proportion of responses are incompatible with the sensory input. This was not the case for the data reported here. Although some responses did not have the correct first phoneme, they 
were compatible (as far as one could determine) with the sensory input. In Salasoo and Pisoni's (in press) study, in which subjects did produce some responses $(5 \%)$ that were incompatible with the sensory input, most of these occurred in the early gates. If one assumes, as do Salasoo and Pisoni, that $150 \mathrm{msec}$ is needed to set up a cohort, these data are not in conflict with the claim. Indeed, on the basis of these data, Salasoo and Pisoni argue in favor of the priority of bottom-up input when at least $150 \mathrm{msec}$ of the beginning of the word has been presented. They propose, in addition, that responses based on nonsensory sources of information are elicited only with very short segments of speech.

Other evidence supporting the claim that the cohort is initially activated by the sensory input is the finding that subjects produced the same number of word candidates in the first gate in all conditions. If context had functioned predictively, allowing the activation of only those word candidates that were contextually appropriate, then we would have observed smaller initial sets of elicited words in the context conditions than in the isolation condition.

\section{Effects of Context}

The data support the claim that context begins to have an effect only after the initial cohort has been generated. At that point, active word candidates start to be assessed for their contextual compatibility and those that are not suitable drop out of the pool. This explains the difference between the isolation condition and the context conditions in the rate with which the size of the cohort is reduced with each successive gate. The relatively steady rate of decrease in the number of word candidates across gates in the isolation condition is the result of the continuous assessment of word candidates against the accumulating sensory input. In the minimal-semantic-context conditions, the rate of decline started earlier and was much faster, accelerating steeply over the first few gates. This reflects the increasingly strong effects of context as it starts to operate on the pool of candidates over the first 100 $250 \mathrm{msec}$ of the word.

This early integration of contextual constraints with the sensory input is contrary to the claim that word recognition is an autonomous process with context having an effect only after a word has been recognized (e.g., Swinney, 1982). For this proposal to be accurate, there should have been no difference in the drop-off curves for segments heard in isolation or in context.

Apart from specifying that context functions after the initial cohort has been generated, the cohort theory has had little to say about the particular ways in which context operates on the cohort. The present data do suggest some constraints on how context might be captured in the model.

If, for example, word candidates are evaluated in an all-or-none manner for their contextual suitability as soon as the cohort is generated (say in the first $150 \mathrm{msec}$ ), unsuitable candidates discarded, and thereafter the remaining candidates assessed only for their compatibility with the incoming sensory input, then there should be no con- textually inappropriate responses after $150 \mathrm{msec}$. The data do not support this account. The reduction in the size of the cohort over time was clearly different, depending upon whether a word was heard in isolation, a meaningful context, or an anomalous context. Although there was a steady attrition rate for words in isolation, for words in a meaningful context, a large number of word candidates dropped out of the pool during the first $200-250 \mathrm{msec}$ and thereafter the drop-off rate slowed down. This suggests that only those candidates that are actually anomalous are immediately rejected from the cohort, and that only those that are implausible in some respect are discarded less readily - and possibly only when the sensory input confirms the choice.

The data also suggest that syntactic, semantic, and prosodic context affects the initial cohort by providing the listener with cues restricting the number of syllables in the target word. Although the precise nature of these cues and the ways in which they are deployed remains unclear, this is an aspect of contextual constraint that ultimately needs to be taken into account in the cohort theory.

Finally, these and other results suggest that effects of word frequency can be observed in the early stages of the word recognition process. Such effects have not as yet been incorporated into the cohort theory, nor is it apparent how they should be. Several experiments demonstrate effects of word frequency, but the exact nature of the effect remains unclear. In the gating task, for example, subjects do not routinely produce the highest frequency members of the cohort. Although more words of high frequency were produced than words of either low or medium frequency, words covering the entire frequency range were elicited. If there was a simple relationship between frequency and, for example, level of activation of ease of access, then subjects should have consistently produced the highest frequency members of the initial set. Moreover, frequency effects disappeared $200 \mathrm{msec}$ into the word. On the basis of these gating data, then, it is difficult to determine the role that frequency plays in spoken word recognition and, consequently, to decide how frequency should be captured in the model.

In conclusion, this kind of analysis of elicited responses provides details about the structure of the cohort which are both informative and difficult to obtain by other means. Moreover, the fact that the gating task produces data that are consistent with a model developed on the basis of very different tasks suggests that it is an appropriate tool for investigating the kinds of issues raised in this paper.

\section{REFERENCES}

Amerman, J., \& Parnell, M. (1981). Influence of context and rate of speech on stop-consonant recognition. Journal of Phonetics, 9 , 323-332.

BradLEy, D. (1978). Computational distinctions of vocabulary type. Unpublished doctoral dissertation M.I.T

Cotton, S., \& Grosjean, F. (1984). The gating paradigm: A comparison of successive and individual presentation formats. Perception \& Psychophysics, 35, 41-48. 
Forster, K. (1976). Accessing the mental lexicon. In R. Wales \& E. C. T. Walker (Eds.), New approaches to language mechanisms. Amsterdam: North-Holland.

Forster, K. (1981). Priming and the effects of sentence and lexical contexts on naming times: Evidence for autonomous lexical processing. Quarterly Joumal of Experimental Psychology, 33A, 465-495.

Foss, D. (1969). Decision processes during sentence comprehension: Effects of lexical item difficulty and position upon decision times. Journal of Verbal Learning and Verbal Behavior, 8, 457-462.

Glanzer, M., \& EhrEnREICH, S. (1980). Structure and search of the internal lexicon. Journal of Verbal Learning and Verbal Behavior, 18, 381-398.

Gordon, B. (1983). Lexical access and lexical decision: Mechanisms of frequency sensitivity. Journal of Verbal Learning and Verbal Behavior, 22, 24-44.

Grosjean, F. (1980). Spoken word recognition processes and the gating paradigm. Perception \& Psychophysics, 28, 267-283.

LANDAUER, T., \& STREETER, L. (1973). Structural differences between common and rare words: Failure of equivalence assumptions for theories of word recognition. Journal of Verbal Learning and Verbal Behavior, 60, 119-131.

MARSLEN-WILSON, W. D. (1984). Parallel processes in lexical access. Unpublished manuscript, University of Cambridge.

MARSLEN-Wilson, W. D. (1980). Speech understanding as a psychological process. In J. C. Simon (Ed.), Spoken language generation and understanding. Dordrecht: Reidel.

MARSLEN-WILSON, W. D. (1984). Function and process in spoken wordrecognition. In H. Bouma \& D. G. Bouwhuis (Eds.), Attention and performance: Control of language processes. Hillsdale, NJ: Erlbaum.

MARSLEN-WILSON, W. D., \& TYLER, L. K. (1980). The temporal structure of spoken language understanding. Cognition, 8, 1-71.

Marslen-Wilson, W. D., \& Welsh, A. (1978). Processing interactions and lexical access during word-recognition in continuous speech. Cognitive Psychology, 10, 29-63.

McCusker, L., Holley-Wilcox, P., \& Hillinger, M. (1979, April). Frequency effects in auditory and visual word recognition. Paper presented at the 25th Annual convention of the Southwestern Psychological Association, San Antonio, Texas.

MorTOn, J. (1969). Interaction of information in word recognition. Psychological Review, 76, 165-178.

Nooteboom, S., \& Truin, P. G. M. (1980). Word recognition from fragments of spoken words by native and non-native listeners (IPO Annual Progress Report No. 15). Eindhoven, The Netherlands: Institute for Perception Research.

SALASOO, A., \& PISONI, D. (in press). Interaction of knowledge sources in spoken word identification. Journal of Verbal Learning and Verbal Behavior.

SeidenberG, M., Tanenhaus, M., Leiman, J., \& Bienkowski, M. (1982). Automatic access of the meanings of ambiguous words in context: Some limitations of knowledge-based processing. Cognitive Psychology, 14, 489-537.

ShIElDs, J., McHugh, A., \& MARTIN, J. (1974). Reaction time to phoneme targets as a function of rhythmic cues in continuous speech. Journal of Experimental Psychology, 102, 250-255.

SWINNEY, D. (1979). Lexical access during sentence comprehension: (Re)consideration of context effects. Journal of Verbal Learning and Verbal Behavior, 18, 645-659.

SwINNEY, D. (1982). The structure and time-course of information interaction during speech comprehension: Lexical segmentation, access and interpretation. In J. Mehler, E. C. T. Walker, \& M. F. Garrett (Eds.), Perspectives on mental representation. Hillsdale, NJ: Erlbaum.
TYLER, L. K., \& WESSELS, J. (1983). Quantifying contextual contributions to word recognition processes. Perception \& Psychophysics, 34, 409-420.

Tyler, L. K., \& Wessels, J. (1984). Is gating an on-line task: Evidence from naming latency data. Unpublished manuscript, MPI fur Psycholinguistic, Nijmegen, The Netherlands.

UIT DEN BoOgaART, P. C. (1975). Woordfrequenties in geschrieven en gesproken Nederlande. Utrecht: Oosthoek, Scheltema \& Holkema.

WHALEY, C. P. (1978). Word-nonword classification time. Journal of Verbal Learning and Verbal Behavior, 17, 143-154.

\section{NOTES}

1. This estimate of the minimum amount of sensory input required to initiate a cohort is based upon measurements of word-recognition times obtained from a variety of word monitoring and shadowing studies.

2. Targets in this study ranged in frequency from 4 to 64 per 720,000 (Uit den Boogaart, 1975).

3. These estimates were based on dictionary entries.

4. Literal English gloss. The syntactic constraints do not function the same way in English as they do in Dutch.

Target: To benefit

(a) The appointment with the dentist goes not through. John tries to ....

(b) The appointment with the dentist goes not through. John can . . .

(c) The breath with the lie shuffles only through. The terrace tries to...

(d) The breath with the lie shuffles only through. The terrace will ....

5. Only those 17 items were analyzed in which we had established (Tyler \& Wessels, 1983) that /te/ was perceived by listeners and therefore provided the appropriate strong syntactic constraints.

6. Predictability was measured differently in the two experiments. In both cases, a cloze test was used, but the present study also included the first $100 \mathrm{msec}$ of each target. This restricted the set of words likely to be produced, and provided a more conservative test of predictability.

7. The two minimal semantic conditions are not reported separately, since an ANOVA showed no difference between them $(F<1)$.

8. The minimal vs. anomalous semantic contexts are reported separately because they were significantly different from each other on an ANOVA $[\mathrm{F}(3,186)=4.51, \mathrm{p}<.01]$.

9. Subjects did not produce a large proportion of extremely highfrequency words. The median frequency of the elicited responses at the first gate was between 30 and 35 (depending on condition).

10. This notion of activation threshold contrasts with Morton's (1969) use of decision threshold in the logogen model.

11. Thanks to an anonymous reviewer for pointing this out to me.

12. Sixty-five percent of target words elicited at least one inappropriate response in the first gate in the anomalous/strong-syntax condition. This compares with $38 \%$ in the minimal-semantic/strong-syntax condition, $35 \%$ in the anomalous/weak-syntax condition, and only $6 \%$ in the minimal-semantic/weak-syntax condition.

13. Only $25 \%$ of targets elicited any semantically inappropriate responses at all in the first gate. 
APPENDIX

Percentage of Elicited Words of 1, 2, 3, or 4 Syllables in Length in the Isolation, Anomalous, and Minimal Semantic Context Conditions, at Gates 2-5, as a Function of Number of Syllables in the Target

\begin{tabular}{|c|c|c|c|c|c|c|c|c|c|c|c|c|c|c|c|c|}
\hline \multirow[b]{3}{*}{ Context } & \multicolumn{4}{|c|}{ Gate 2} & \multicolumn{4}{|c|}{ Gate 3} & \multicolumn{4}{|c|}{ Gate 4} & \multicolumn{4}{|c|}{ Gate 5} \\
\hline & \multicolumn{12}{|c|}{ Number of Syllables } & \multirow[b]{2}{*}{1} & \multirow[b]{2}{*}{2} & \multirow[b]{2}{*}{3} & \multirow[b]{2}{*}{4} \\
\hline & 1 & 2 & 3 & 4 & 1 & 2 & 3 & 4 & 1 & 2 & 3 & 4 & & & & \\
\hline \multicolumn{17}{|c|}{ Two-Syllable Targets } \\
\hline Isolation & 64 & 34 & 2 & 0 & 60 & 37 & 3 & 0 & 61 & 38 & 1 & 0 & 56 & 40 & 4 & 0 \\
\hline Anomalous & 38 & 42 & 12 & 0 & 38 & 55 & 6 & 1 & 36 & 61 & 3 & 1 & 36 & 63 & 2 & 0 \\
\hline Minimal Semantic & 34 & 56 & 9 & 2 & 31 & 58 & 9 & 3 & 25 & 64 & 8 & 4 & 20 & 72 & 6 & 3 \\
\hline \multicolumn{17}{|c|}{ Three-Syllable Targets } \\
\hline Isolation & 36 & 46 & 16 & 2 & 28 & 49 & 21 & 2 & 27 & 44 & 29 & 0 & 17 & 41 & 41 & 1 \\
\hline Anomalous & 18 & 39 & 37 & 7 & 13 & 41 & 39 & 8 & 4 & 42 & 43 & 12 & 1 & 34 & 59 & 6 \\
\hline Minimal Semantic & 17 & 21 & 58 & 5 & 8 & 26 & 57 & 8 & 8 & 24 & 61 & 6 & 2 & 20 & 69 & 10 \\
\hline \multicolumn{17}{|c|}{ Four-Syllable Targets } \\
\hline Isolation & 42 & 35 & 20 & 3 & 11 & 45 & 33 & 11 & 9 & 39 & 30 & 21 & 9 & 23 & 26 & 42 \\
\hline Anomalous & 16 & 28 & 47 & 10 & 2 & 23 & 53 & 22 & 2 & 22 & 47 & 31 & 1 & 16 & 31 & 52 \\
\hline Minimal Semantic & 5 & 19 & 55 & 22 & 2 & 13 & 48 & 38 & 0 & 10 & 38 & 52 & 1 & 6 & 25 & 68 \\
\hline
\end{tabular}

(Manuscript received November 29, 1983;

revision accepted for publication August 6, 1984.) 